\title{
Evaluation of Neuroprotection and Antioxidant Activities via Drosophila Model System in the Active Principle Derived from Sida Glutinosa Comm. Ex Cav. - An Aboriginal Ethno-Medicinal Plant Drug Practiced in the Folklore Medicinal System
}

\author{
Panduranga Murthy G1*, Leelaja BC ${ }^{2}$, Ravishankar HG $^{3}$, Dharshan Raj CG ${ }^{4}$ and Rajesh Kumar \\ ${ }^{1}$ Maharaja Institute of Technology Thandavapura-571 302, Visvesvaraya Technological University, Belagavi, India \\ ${ }^{2}$ DOS in Molecular Biology, University of Mysore, Mysore-570 006, India
}

${ }^{3}$ Hamsageetha Research Foundation, Tumkur-572 102, India

${ }^{4}$ PA College of Engineering, Mangalore - 574 153, Karnataka, India

${ }^{5}$ University Department of Chemistry, Ranchi University, Ranchi-834 001, India

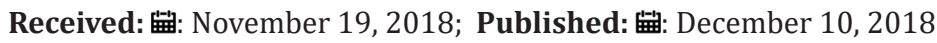

*Corresponding author: Panduranga Murthy G, Maharaja Institute of Technology Thandavapura, India

\begin{abstract}
Sida glutinosa Comm. ex Cav.- An aboriginal ethno-medicinal plant drug belongs to family Malvaceae, commonly called as 'Sticky Fanpetals'. It is a sub-shrub which is available in the forest at shady areas along Ravines. The plant was collected at Biligirirangana Hills (B.R. Hills) of Chamarajanagar district, Karnataka, India and taxonomically identified. The therapeutic information was recorded from traditional medicine men during interaction with tribal healers at B.R. Hills. In the study, methanol extract of Sida glutinosa (G) was preliminarily screened for Physico-chemical and Phytochemical properties. Further, the antioxidant activity both in vitro and in vivo was carried-out. The neuroprotective study was explicitly carried out using a model system, Drosophila melanogaster (Oeragon $K$ ) strain adult male flies. The result reveals that, the extract G exhibited concentration dependent DPPH scavenging activity. The oxidative stress markers employed to access in vivo antioxidant property of G included lipid peroxidation products malondialdehyde (MDA) and hydroperoxide (HP), reduced glutathione (GSH). The modulatory effect of G on superoxide dismutase (SOD) and catalase (CAT) was also determined. The oxidative stress was induced by using paraquat at $15 \mathrm{~mm}$. The concentration of extract for studies was fixed based on LC50 values. There was a significant annihilation in the levels of MDA and HP in case co exposure of G with Par treated flies' homogenate. Finally, the level of SOD and CAT was brought to near basal level in the homogenate of flies co exposed with G and Par. In negative geotaxis assay; it was found that, $\mathrm{G}$ was able to rescue the flies significantly from deteriorating locomotors dysfunctions. The extract $\mathrm{G}$ also exhibited significant antibacterial property against the tested strains.

Abbreviations: MDA: Malondialdehyde; HP: Hydroperoxide; GSH: Glutathione; SOD: Superoxide dismutase; CAT: Catalase; ROS : Reactive Oxygen Species; RNS: Reactive Nitrogen Species; PD: Parkinson's Disease; SCZ: Schizophrenia; AD: Alzheimer's Disease; NADPH: Nicotinamide Adenine Dinucleotide Phosphate; DPPH: 1,1-diphenyl-2-picryl-hydrazyl; TBA: thiobarbituric acid; SDS: Sodium lauryl sulphate; OPT: o-phthalaldehyde; TEMED : N,N,N,N Tetramethyl ethylenediamine; EDTA: Ethylenediamine tetra acetic acid; ANOVA: One-Way Analysis Of Variance; MIC: Minimum Inhibitory Concentration
\end{abstract}

\section{Introduction}

Aerobic organisms produce several reactive free radicals continuously in cells during respiration, metabolism and phagocytosis. The free radicals formed within the cells can induce multiple chemical changes in cellular components like membrane lipids, DNA and proteins, which can eventually lead to cell death. The collective terms "Reactive Oxygen Species (ROS) and Reactive Nitrogen Species (RNS)" refer to a variety of free radicals such as superoxide, hydroxyl, peroxyl, nitric oxide, nitrogen dioxide radicals 
as well as for nonradical reactive intermediates like hydrogen peroxide $\left(\mathrm{H}_{2} \mathrm{O}_{2}\right)$ and peroxynitrite (ONOO.) etc. Their excessive production, termed as "Oxidative Stress" has been implicated in many pathological disorders like heart disease, cancer and ageing [1]. Oxidative stress is a harmful condition that occurs when there is an excess of free radicals or a decrease in antioxidant levels. The evidence to date for oxidative stress in Parkinson's Disease (PD), Schizophrenia (SCZ), Alzheimer's Disease (AD) and other neurodegenerative diseases is strongly persuasive. Clinical studies showed that several events associated with Alzheimer's are capable of stimulating production of free radicals and depletion of antioxidant levels. As pointed out, whether oxidative stress is eventually proven to be primary or secondary in etiologic progression, the therapeutic rewards of antioxidants are likely to be substantial. Clearly, strategies aimed at limiting free radical induced oxidative stress and damage may slow the progression of neurodegenerative diseases [2].

Paraquat (1,1-dimethyl-4,4-bipyridynium dichloride) is a quaternary nitrogen herbicide and highly toxic substance for humans and animals; many cases of acute poisoning and death have been reported [3]. The toxicity of paraquat is due to the generation of the superoxide anion which can lead to the synthesis of more toxic Reactive Oxygen Species (ROS) such as hydroxyl radicals and hydrogen peroxide [4]. On the other hand, the oxidation of reduced Nicotinamide Adenine Dinucleotide Phosphate (NADPH) because of paraquat administration results in the disruption of biochemical processes requiring NADPH [5]. The use of antioxidant compounds provides an easy and convenient way of testing the validity of the free radical theory using Drosophila melanogaster as model organism. The organisms are easier to culture and manipulate under laboratory conditions than the mammals. Administration of the test compounds can be done easily by adding the dissolved compounds to the food medium thus ensuring their uptake by the flies [6].

Sida glutinosa is a sub-shrub which is available in the forest in shady areas along ravines. Native of tropical America and naturalized in Asia. It belongs to family Malvaceae, commonly called as 'Sticky Fanpetals' [7]. Roots and stems of the plant contain ephedrine, an important alkaloid. Besides this, traces of sitosterol and palmic, stearic etc. have also been isolated from this plant. It was reported that main alkaloid present in sida is asparagin. Besides this fatty oil, phytosterol, mucin, potassium nitrate, resins and acids are also known to be found in the plant extract. It is also reported that sida does not contain any tannin or glycoside. The recent studies reported that, ephedrine and y-ephedrine are major alkaloids found in the aerial parts of the plant. Besides these two, some other chemical compounds were isolated from the aerial parts of sida are 6-phenyl ethyl amine, carboxylated tryptomines, qunazoline, hypaphorine, vasicinol etc. Different species of this plant have been reported to contain cryptolepine also [8]. It was reported that the extract of sida was useful in the treatment of fevers, ophthalmia, rheumatism, leucorrhoea, micturition, gonorrhea, colic, neurological disorders, analgesic, anti-inflammatory and general impediment. The extract of roots of sida plant was exhibited wound healing property [9].

The plant extracts of Sida glutinosa was used in soliga triabal drug formulations. It was used to cure diarrhea, stimulating \& nutritive. The rejuvenating action of this herb extends to the nervous, circulatory and urinary systems. It was reported that it has diuretic effect and is useful in the treatment of urinary problems, including cystitis. It is used as anti-inflammatory and in treatment of infections and bleeding disorders. Considering medicinal properties reported by the researchers and usage of the plant in the soliga tribal drug formulations, we worked towards a preliminary appraisal of physico-chemical, Phyto-chemical properties, antimicrobial, in vitro, in vivo antioxidant activity and neuroprotection efficacy of leaf extract of Sida glutinosa was carried out for the first time.

\section{Materials and Method}

\section{Plant Material}

A less known ethnomedicinal plant Sida glutinosa was collected at Biligirirangana Hills, Karnataka, India. The plant material was identified taxonomically, and the validation was made by Dr. G. Panduranga Murthy, Scientist and Head, CSRF, Tumkur, India (BRT/024/2008). The information on therapeutic uses was recorded during interaction with tribal (Soliga) medicine men. The plant leaves were collected and used for further studies (Figure 1).

Preparation of the Methanol Extracts: The leaves were collected and rinsed with distilled water. They were dried for 5 days in the dark at room temperature. After five days they were ovendried for 1 hour at $60^{\circ} \mathrm{C}$. They were made into fine powder. Crude extract was prepared in the methanol. The extract was filtered through a Whatman, No. 1 filter paper. The extract was evaporated under reduced pressure using rotary evaporator. The powder was stored in dark glass bottles for further use.

Physico Chemical Characterization: Powder of leaves of Sida glutinosa were subjected to physicochemical study for determination of foreign organic matter, moisture content, Total ash value, Acid insoluble ash, Water soluble ash, using the method described by Indian Herbal Pharmacopeia and United State Pharmacopeia [10].

Preliminary Phytochemical Analysis: Phyto-chemical screening of the Methanol extract of sida glutinosa was carried out to identify the major constituents in the sample. The standard procedure was used to determine Phyto-chemical properties $[11,12]$.

Antimicrobial Studies: The extract G was screened for their antifungal activity against Aspergillus niger, Candida albicans. Antibacterial property was evaluated against Staphylococcus aureus, Salmonella typhi, and Bacillus subtilis strains by disc diffusion method. The compounds were dissolved in DMSO and 
antimicrobial activity was determined by serial plate dilution method [13].

\section{In vitro Antioxidant Activity}

DPPH Radical Scavenging Activity: The DPPH assay was carried out according to the standard method with some modifications. The stock solution was prepared by dissolving 24 mg DPPH with $100 \mathrm{~mL}$ methanol and then stored at $-20^{\circ} \mathrm{C}$ until needed. The working solution was obtained by mixing $10 \mathrm{~mL}$ stock solution with $45 \mathrm{~mL}$ methanol to obtain an absorbance of 1.170 .02 units at $517 \mathrm{~nm}$ using the spectrophotometer. The solution of compounds at $5 \mu \mathrm{g} / \mathrm{ml}$ concentration was prepared in ethanol. The different aliquot of stock solution was used for estimation. It was diluted to $4 \mathrm{ml}$ using distilled water. To this $1 \mathrm{ml}$ of 1,1-diphenyl2-picryl-hydrazyl (DPPH) solution in methanol was added. The mixed solution was incubated at room temperature for $30 \mathrm{~min}$. The absorbance of stable DPPH• was read at $517 \mathrm{~nm}$ using UV-vis. spectrophotometer and the remaining DPPH was calculated [14].

Preparation of Wheat Cream Agar Medium and Culturing of Flies: D. melanogaster (Oregon $K$ ) adult males (8-10 days old) were obtained from Drosophila stock center, Department of Studies in Zoology, University of Mysore, Manasagangotri, Mysore, Karnataka, India. The flies were maintained at $22 \pm 1^{\circ} \mathrm{C}$ and $70-80 \%$ relative humidity, fed on a standard wheat cream agar medium seeded with yeast. The medium was prepared according to standard protocol of the media ( $100 \mathrm{~mL}$ ) containing $10 \mathrm{~g}$ wheat flour, $10 \mathrm{~g}$ jaggery, $1 \mathrm{~g}$ agar and $0.75 \mathrm{ml}$ propionic acid (Antifungal Agent) few granules of yeast were added. After 24 hours flies were transferred to fresh media bottles to avoid sticking of flies to media. Whenever required, the flies were exposed to the fumes of diethyl ether in a small airtight glass container for $1 \mathrm{~min}$. for observation under stereo zoom and for other studies.

Preparation of extract for Feeding the Flies: Plant extract (G) was dissolved in ethanol. The $G$ was introduced into the medium at semisolid state and mixed well and could solidify. Adult males (50 no.) were introduced into the vials containing media. Safety Evaluation of $\mathrm{G}$ was dissolved in Et-OH. The toxicity of Et-OH was checked in the medium by rearing the flies in media with and without $100 \mu \mathrm{L}$ Et-OH. Since there was no mortality in the flies reared on medium containing $100 \mu \mathrm{L}$ Et-OH, for all experimental purposes wheat cream agar medium was used with $0.5 \%$ DMSO. Further studies were carried out to find out whether the compounds are causing mortality in the experimental batches. In this set of experiments, the males (test) were fed on a medium containing G 10, and $20 \mu \mathrm{g} / \mathrm{mL}$ concentrations. In each vial $4 \mathrm{~mL}$ of control food or food containing the compounds were added. The vials were closed with cotton stoppers. Lethality due to compounds was monitored by counting dead flies every $24 \mathrm{~h}$ up to 7 days, and data was expressed in terms of percentage mortality [15].

Whole Body Homogenate Preparation: Sodium-phosphate buffer $0.1 \mathrm{M},(\mathrm{pH}$ 7.4) was used for preparing whole body homogenates. Males (30 no.) from control and tested groups were used for this purpose. After homogenizing, the samples were centrifuged at $2500 \mathrm{Xg}$ for $12 \mathrm{~min}$ at $4^{\circ} \mathrm{C}$. The supernatant was filtered through nylon mesh (pore size, $10 \mu \mathrm{m}$ ) and used for biochemical assays [16].

Paraquat Exposure and Concentrations: In a preliminary study, flies were exposed to paraquat at concentrations of 10, 15, 20 and $25 \mathrm{~mm}$ for $96 \mathrm{~h}$ to determine lethality. However, to assess the antioxidant effect of G, only one concentration of Paraquat (15 $\mathrm{mM}$ ) was employed. For these studies, paraquat exposed flies were provided with $\mathrm{G}(20 \mu \mathrm{g} / \mathrm{mL})$ in the diet and we determined the modulatory effect of $\mathrm{G}$ on paraquat induced oxidative impairments and locomotors dysfunction.

Typical Exposure Protocol: For mortality studies, a minimum of 50 adult flies per replicate (three replicates) were exposed to $G$ at $20 \mu \mathrm{g} / \mathrm{mL}$ or Paraquat $15 \mathrm{mM}$ or a combination of G+ Paraquat. Lethality of the flies was monitored for $96 \mathrm{~h}$ and data was expressed in terms of percent mortality.

Paraquat Resistance Test: Two days after emergence from the pupae, male flies were fed with control food or food containing the compounds for a period of 7 days. Then 50 of the flies fed with the test $\mathrm{G}$, and the control were starved for $6 \mathrm{~h}$ to be sure that no food remained in the digestive tract so that none of the compounds would alter the uptake of paraquat. Afterwards, the flies were transferred to vials containing only filter paper soaked with $15 \mathrm{~mm}$ paraquat in $5 \%$ sucrose solution. This concentration of paraquat was selected because it let us to study the flies for a period of $24 \mathrm{~h}$ or more after treatment. Survival was determined 24 and $48 \mathrm{~h}$ later. Survived flies were used for homogenization for biochemical assays. Each assay was repeated thrice [16].

\section{Biochemical Investigations}

Assay for Lipid Peroxidation: Lipid peroxidation was measured by employing Thiobarbituric acid (TBA). Briefly, the reaction mixture containing $500 \mathrm{~mL}$ fly homogenate, $1.5 \mathrm{~mL}$ acetic acid (pH 3.5, 20\%), $1.5 \mathrm{~mL}$ of TBA (0.8\% w/v), $200 \mathrm{~mL}$ sodium lauryl sulphate (SDS) $(8 \% \mathrm{w} / \mathrm{v})$. The mixture was heated in a boiling water bath for $45 \mathrm{~min}$ and adducts formed were extracted into $3 \mathrm{~mL}$ of 1-butanol. The absorbance was measured at $532 \mathrm{~nm}$ and quantified as malondialdehyde equivalents using 1,1,3,3tetramethoxypropane as the standard [17].

Assay for Hydroperoxide: Hydroperoxide generation was determined according to the method of An aliquot of homogenate (100 mg protein) was added to $1 \mathrm{~mL}$ of FOX reagent $(250 \mathrm{mM}$ Ferrous ammonium sulphate; $100 \mathrm{mM}$ sorbitol; $25 \mathrm{mM} \mathrm{H}_{2} \mathrm{SO}_{4} ; 100$ $\mathrm{mM}$ xylenol orange) and incubated for $30 \mathrm{~min}$ at room temperature [18]. The absorbance was taken at $560 \mathrm{~nm}$ and expressed as $\mathrm{nm}$ $\mathrm{HP} / \mathrm{mg}$ protein. 
Estimation of Reduced Glutathione: Reduced glutathione (GSH) content was estimated based on a fluorometric method [19] employing o-phthalaldehyde (OPT). An aliquot of homogenate (stable reduced glutathione with $0.1 \mathrm{M}$ formic acid, $5200 \mathrm{X}$ for 10 $\mathrm{min}$ ) was allowed to react with OPT ( $1 \mathrm{mg} / \mathrm{mL}$ in methanol) at room temperature for $30 \mathrm{~min}$ and fluorescence measured at excitation of $345 \mathrm{~nm}$ and emission at $425 \mathrm{~nm}$.

Activities of Antioxidant Enzymes: Catalase activity was determined according to a reported method. To $1 \mathrm{~mL}$ reaction mixture containing $8.8 \mathrm{mM} \mathrm{H}_{2} \mathrm{O}_{2}(3 \%), 0.1 \mathrm{mM}$ sodium-phosphate buffer, pH 7.0 the reaction was initiated by adding an aliquot (equivalent to $10 \mathrm{mg}$ protein). The decrease in $\mathrm{H} 2 \mathrm{O} 2$ was monitored for $3 \mathrm{~min}$ at $240 \mathrm{~nm}$ [20]. SOD activity was determined by monitoring the inhibition of quercetin auto oxidation. Total volume of $1 \mathrm{~mL}$ reaction mixture containing 3-5 mg protein; $0.016 \mathrm{M}$ sodium phosphate buffer, pH 7.8; N,N,N,N Tetramethyl ethylenediamine (TEMED), 8mM and Ethylenediamine tetra acetic acid (EDTA), $0.08 \mathrm{mM}$ and reaction was started by adding $0.15 \%$ quercetin dissolved in dimethyl formamide. Reaction was monitored for 3 $\min$ at $406 \mathrm{~nm}$, expressed as amount of protein required to inhibit $50 \%$ of quercetin auto oxidation [21]. Protein estimation was done using flies' homogenate [22].

Negative Geotaxis Assay: Test flies were anesthetized and placed in a vertical glass column (length, $25 \mathrm{~cm}$; diameter, $1.5 \mathrm{~cm}$ ) sealed at one end. After a brief recovery period, flies were gently tapped to the bottom of the column. Following $1 \mathrm{~min}$, flies that reached to the top of the column and flies that remained at the bottom were counted separately. Data was expressed as percent flies escaped beyond minimum distance of $6 \mathrm{~cm}$ in $60 \mathrm{~s}$ of interval $[23,24]$. Per replication 20 adults were used for each assay and the assay was repeated three times. The score for each replication was an average of three such trials for each group including control.

\section{Statistical Analysis}

Data were expressed as mean SD and all statistical analysis was performed using Prism 3.0 software Comparisons were performed with the One-Way Analysis Of Variance (ANOVA) test. All experiments were performed in triplicates.

\section{Results and Discussion}

The methanol extract $\mathrm{G}$ was characterized physic chemically and phytochemical analysis the data is illustrated in the (Tables 1 \& 2) respectively. The results reveal that the extract is associated with flavonoids, phenols and other components. The extract $\mathrm{G}$ was also screened for their antibacterial activity against Escherichia coli K-12, Staphylococcus aureus, Salmonella typhi, and Bacillus subtilis. It was also screened for their antifungal activity against Aspergillus Niger and Candida albicans. The extract G exhibited significant activity against tested organisms However, based on this promising observation, it is immature to arrive at the conclusion. The zone of inhibition and the Minimum Inhibitory Concentration (MIC) values of the compounds tested against bacteria and fungi are given in the (Table 3). The in vitro antioxidant assays like DPPH and TBARS assay were carried out. The extract $G$ exhibited better antioxidant activity in concentration dependent manner. The data is depicted in the (Figures $2 \& 3$ ) respectively.

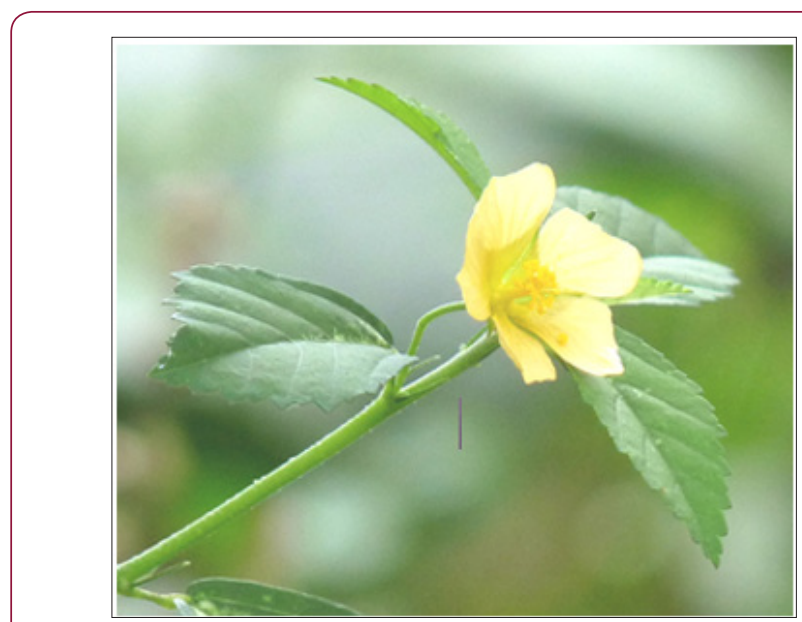

Figure 1: Sida glutinosa- A natural habitat.

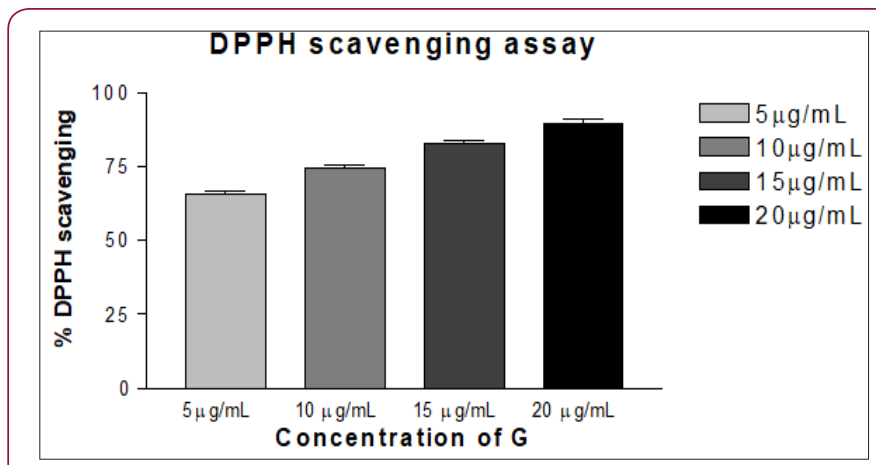

Figure 2: In vitro DPPH scavenging activity of methanol extract of Sida glutinosa (G). Result is expressed as \% $\mathrm{DPPH} \bullet$ scavenged.

Table 1: Physico chemical Characterizations of methanol extract of Sida glutinosa.

\begin{tabular}{|c|c|c|}
\hline Sl.no & Parameters & Average value $\mathbf{\%} \mathbf{w} / \mathbf{w}$ \\
\hline 1 & Total ash & 34.2 \\
\hline 2 & Acid insoluble ash & 52.046 \\
\hline 3 & Water soluble & 75.438 \\
\hline 4 & Foreign organic matter & 6.7 \\
\hline
\end{tabular}

Table 2: Phyto-chemical analysis of methanol extract of Sida glutinosa..

\begin{tabular}{|c|c|c|}
\hline Sl. No & Class of Constituents & Remarks \\
\hline 1 & Carbohydrates & + \\
\hline 2 & Proteins & + \\
\hline 3 & Phenolic compounds & - \\
\hline 4 & Saponins & + \\
\hline 5 & Flavonoids & - \\
\hline 6 & Alkaloids & + \\
\hline
\end{tabular}


Table 3: Antimicrobial activity of G.

\begin{tabular}{|c|c|c|c|c|}
\hline Extract & Staphylococcus aureus & Bacillus subtilis & Salmonella typhi & Escherichia coli \\
\hline G & $29(20)$ & $30(20)$ & $27(30)$ & $29(10)$ \\
\hline Standard & 24 & 23 & 23 & 25 \\
\hline DMSO & 0 & 0 & 0 & 0 \\
\hline
\end{tabular}

Note: Standard drug used: Bacteria (Ciprofloxacin).

G used

( $40 \mu \mathrm{g}$ in $10 \mathrm{~mL}$ - based on MIC concentration).

Control DMSO (dimethyl sulphoxide)

Zone of Inhibition in $\mathrm{mm} \quad$ :

MIC in $\mu \mathrm{g} / \mathrm{mL}$ (data given in the parenthesis is for MIC).

The key objective was to elucidate antioxidant and preliminary neuro-protective efficacy of the $\mathrm{G}$ in vivo. The model organism,. Drosophila melanogaster was used in this study because it is an excellent model for gerontological research due to its relatively short life span; the adult flies appear to show many of the manifestations of cellular senescence observed in mammals and oxidative stress plays an important role in governing the life span of the fly. Toxicity study was done to determine the lethal concentration of G. Safety evaluation of $\mathrm{G}$ revealed that the $\mathrm{G}$ was nontoxic at experimental concentration which is given in (Table 4).
Different concentrations of Paraquat were used to determine concentration and time dependent mortality of flies. Concentration and time dependent mortality response of paraquat on adult male Drosophila melanogaster was given in the (Figure 4). Result reveals that, there was high mortality (50-60\%) among flies during a 7-day exposure period with Par. Where as in case of co exposure of $G$ and Par showed significant reduction in mortality rate. The solvent ethanol $(100 \mu \mathrm{l})$ was kept as solvent control. In this case also mortality rate is insignificant compared to Par treated flies.

Table 4: Lethality Test: Expressed in Percent mortality in Normal flies (Control), Et-OH (Solvent) G at 10,20,40 $\mu$ g/ml concentrations.

\begin{tabular}{|c|c|c|c|c|c|c|c|c|}
\hline & Concentration & 1 & 2 & 3 & 4 & 5 & 6 & 7 Days \\
\hline $\begin{array}{l}\text { Standard } \\
\text { media }\end{array}$ & --- & 0.0 & 0.0 & $0.26 \pm 0.05$ & $0.26 \pm 0.05$ & $0.16 \pm 0.05$ & $0.23 \pm 0.05$ & $0.33 \pm 0.05$ \\
\hline Et-OH $(\mu \mathrm{l})$ & 100 & $0.766 \pm 0.32$ & $0.66 \pm 0.25$ & $1.6 \pm 0.31$ & $2.03 \pm 0.20$ & $1.76 \pm 0.20$ & $1.93 \pm 0.20$ & $2.4 \pm 0.26$ \\
\hline $\mathrm{G}(\mu \mathrm{g} / \mathrm{ml})$ & 10 & $0.46 \pm 0.25$ & $0.56 \pm 0.40$ & $0.63 \pm 0.36$ & $0.83 \pm 0.36$ & $1.46 \pm 0.21$ & $2.1 \pm 0.19$ & $1.83 \pm 0.37$ \\
\hline
\end{tabular}

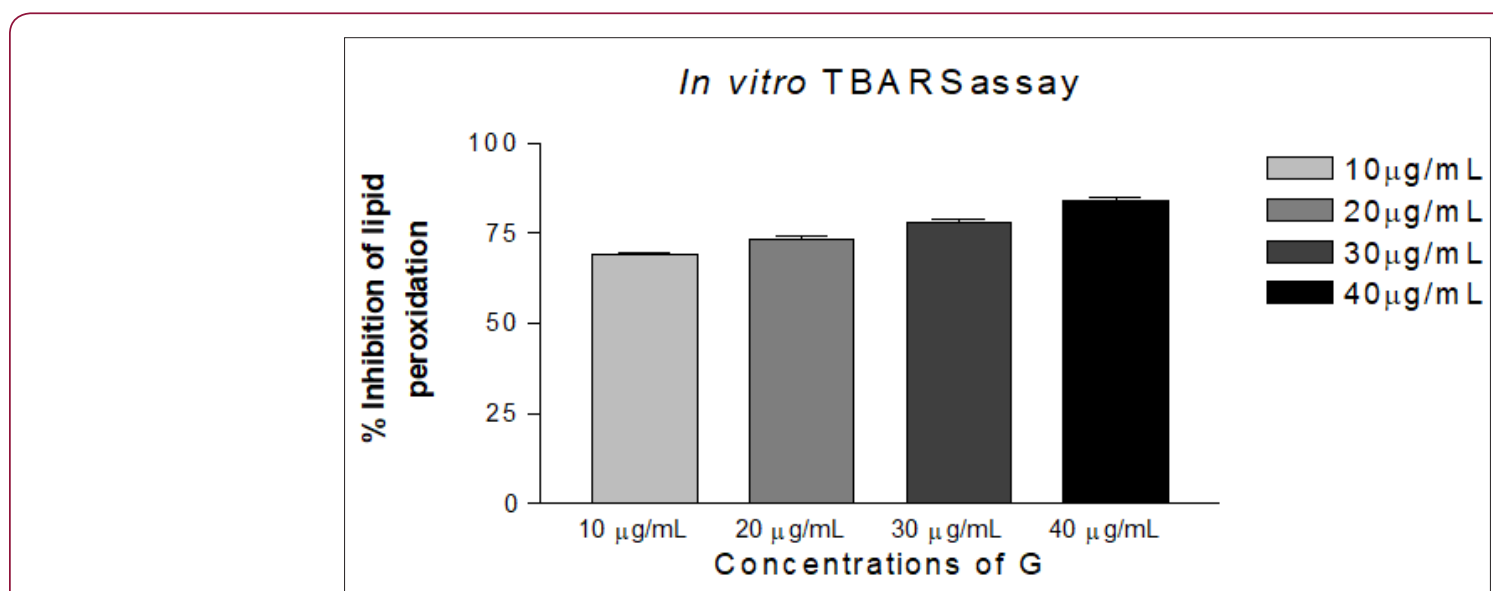

Figure 3: In vitro lipid peroxidation assay for G.

It was known that nigrostriatal dopaminergic neurons are very sensitive to the toxicity of paraquat which can also be toxic to glial cells [25-27]. To determine neuroprotective efficacy of the G geotaxis assay was carried out. Accordingly, the locomotor deficits among flies of various treatment groups employing a similar paradigm. The Paraquat treated flies exhibited severe locomotor impairments as evident by the large number of flies staying at the bottom of the glass column, while co-treatment with G significantly improved the performances of flies. With G treatment, a greater number of flies (50-60\%) showed negative geotaxis behavior clearly indicating its neuroprotective effect. The data is showed in the (Figure 4). Incidence of mortality among adult male Drosophila melanogaster exposed to paraquat, is considerably high whereas there was a decrease incidence of mortality among flies exposed to $\mathrm{G}+$ PAR, This results support that $\mathrm{G}$ showed protective action. The data is given in the (Figure 6). The above results instigated us to determine antioxidant effect of $\mathrm{G}$ against paraquat induced oxidative stress. 


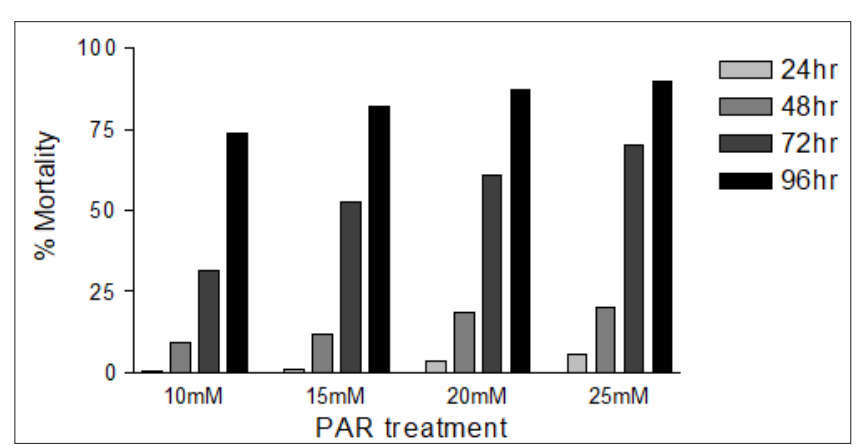

Figure 4: Concentration and time dependent mortality response among adult male Drosophila melanogaster exposed to PAR in the feed. $(n=50$ flies per replicate, three such replication used for assay).

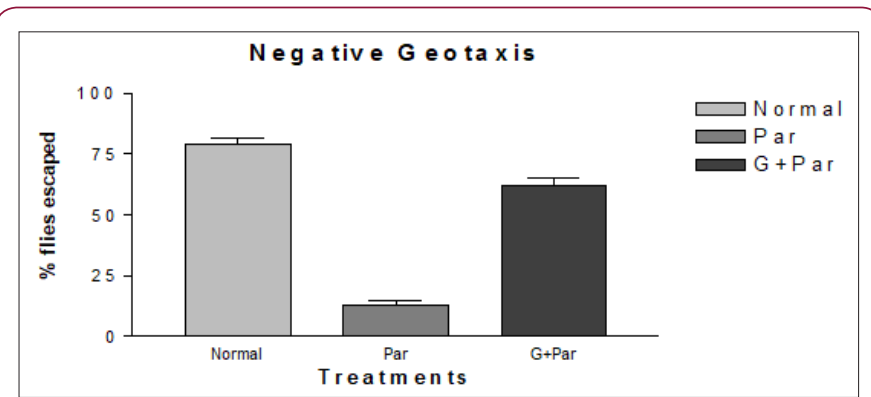

Figure 5: Modulation of paraquat induced locomotor (expressed as percent flies escaped) deficits among adult male Drosophila melanogaster by $\mathrm{G}(\mathrm{n}=50$ flies per replicate, three such replication used for assay. data was analyzed by one-way ANOVA followed by post hoc Tukey test ( ${ }^{*} \mathrm{p}$ $<0.05)$.

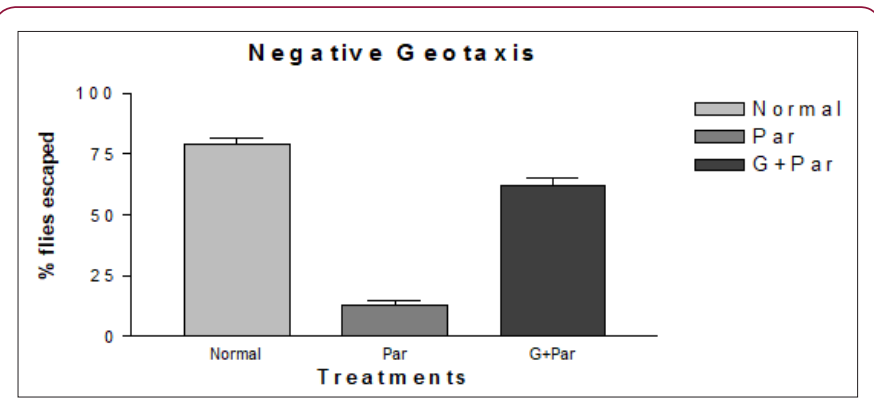

Figure 6: Incidence of mortality among adult male Drosophila melanogaster exposed to Paraquat, co-exposure as G+ PAR and solvent Ethanol (Et-OH); note: decrease incidence of mortality among flies co-exposed with $\mathrm{G}^{+}$ PAR . Values are mean \pm SE (in triplicates), data was analyzed by one-way ANOVA followed by post hoc Tukey test $\left({ }^{*} p<0.05\right)$. Significances were determined by making comparisons between control vs. G, PAR*.

It was reported that significant induction of oxidative stress among flies exposed to paraquat $15 \mathrm{mM}$ evidenced by the marked elevation in MDA and hydroperoxide level coupled with significant change in the activities of antioxidant enzymes such as CAT, SOD which suggested an increased generation of ROS. Ameliorative effects of $G$ on paraquat induced oxidative stress markers in whole body homogenates of adult flies fed with G supplemented diet for
7 days showed significant diminution in MDA and HP levels. The data is given in (Figures $7 \&$ 8) respectively. The reduced GSH is a tripeptide and most abundant soluble antioxidant molecule which plays a crucial role in detoxifying ROS either by reacting directly with radicals non-enzymatically or acting as a substrate in GST catalyzed reactions. Severe depletion in cellular GSH levels upon paraquat exposure in Drosophila adds further evidence that a state of oxidative stress exists in vivo which may lead to mitochondrial damage, increase in free radical generation and peroxidation of membrane lipids. Although paraquat exposure for 7 days caused significant decrease in GSH level, on co exposure with G, flies were able to restore the depleted GSH levels significantly (Figure 9) clearly suggesting the ability of G to up regulate levels of GSH.

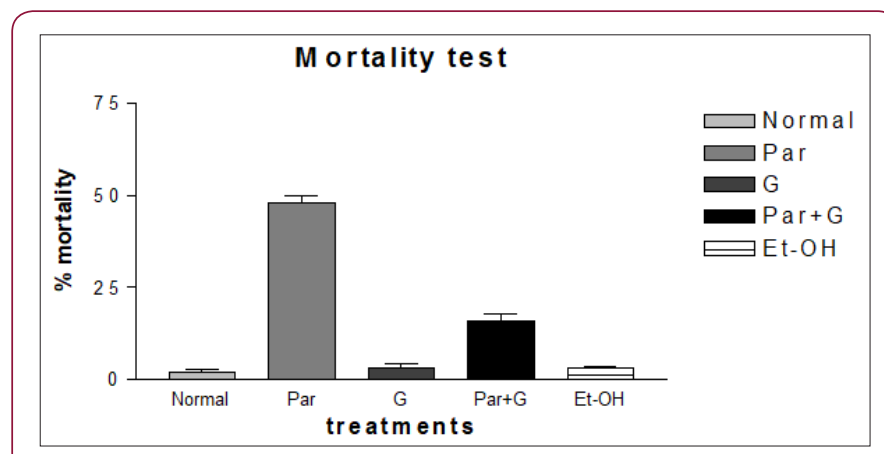

Figure 7: Incidence of mortality among adult male Drosophila melanogaster exposed to Paraquat, co-exposure as G+ PAR and solvent Ethanol (Et-OH); note: decrease incidence of mortality among flies co-exposed with G+ PAR. Values are mean \pm SE (in triplicates), data was analyzed by one-way ANOVA followed by post hoc Tukey test $\left({ }^{*} \mathrm{p}<0.05\right)$. Significances were determined by making comparisons between control vs. G, PAR*.

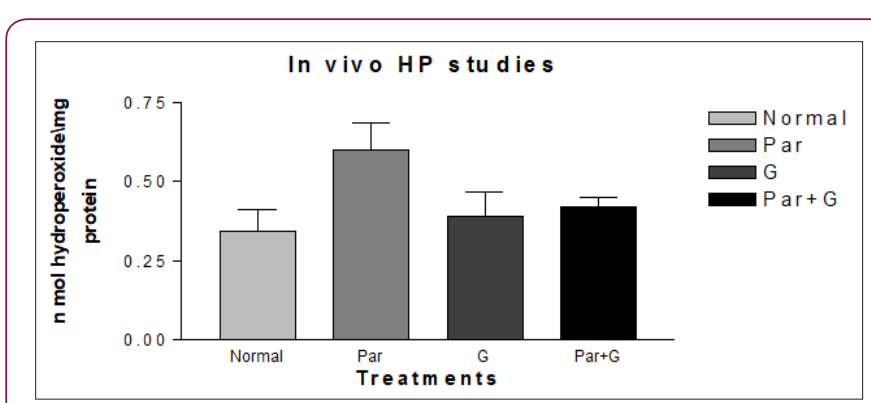

Figure 8: Ameliorative effect of $G$ treatment on Paraquat (PAR) induced oxidative stress measured as hydroperoxide levels in whole body homogenates of adult Drosophila melanogaster. Where $\left(\mathrm{p}^{*}<0.05\right.$,) for the groups treated with Par+ $\mathrm{G}^{*}$ compared with Par.

Paraquat(15 mM) exposure induced a significant elevation in the activity of all antioxidant enzymes measured. However, among $\mathrm{G}+$ paraquat exposed flies, the activity levels of CAT and SOD, were restored to near basal levels, (Figures 10 \& 11). Based on biochemical evidences, we propose that dietary feeding of leaf extract of Sida glutinosa (G) to Drosophila for a short duration has the propensity to attenuate paraquat induced oxidative stress owing to its antioxidative nature and its ability to modulate the activities 
of antioxidant defenses such as reduced GSH and antioxidant defenses. Additional evidences viz., lower incidence of paraquat induced mortality and higher resistance to paraquat among flies pretreated with G. Further, its antioxidant property was clear by its ability to significantly abrogate paraquat induced oxidative stress, by depleting the lipid peroxidation product malondialdehyde.

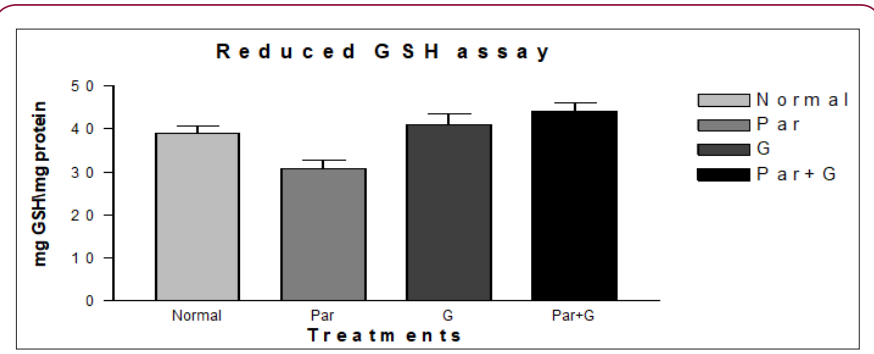

Figure 9: Ameliorative effect of $G$ treatment on Paraquat (PAR) induced oxidative stress measured as reduced glutathione levels in whole body homogenates of adult Drosophila melanogaster. Where $\left(\mathrm{p}^{*}<0.05\right.$, ) for the groups treated with $G^{*}$ and Par $+G^{*}$ in comparison with normal and Par.

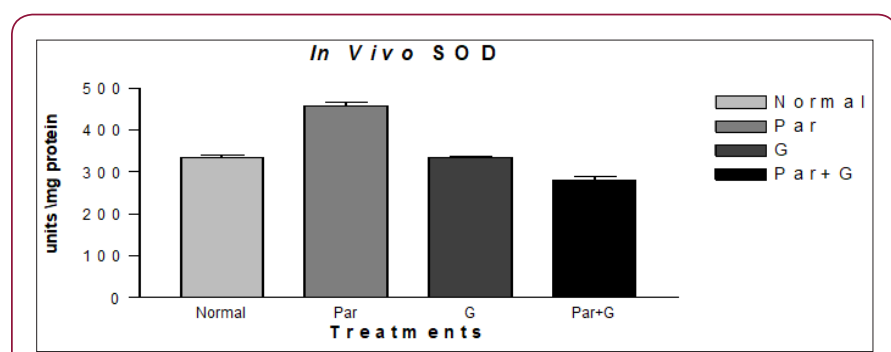

Figure 10: Modulatory effect $G$ on the endogenous markers of oxidative stress HP in adult Drosophila melanogaster. Significances were determined by making comparisons between control vs treated group. Where $\left({ }^{*} p<0.05\right)$ for the groups treated for $G^{*}$ with the control group and Par+ $\mathrm{G}^{*}$ with Par. Modulatory effect of $\mathrm{G}$ on the endogenous markers of oxidative stress SOD in adult Drosophila melanogaster. No significant change in the level of SOD found the groups treated with $G$ but restored to near basal level compared to control and Par+ $G^{*}$ with Par.

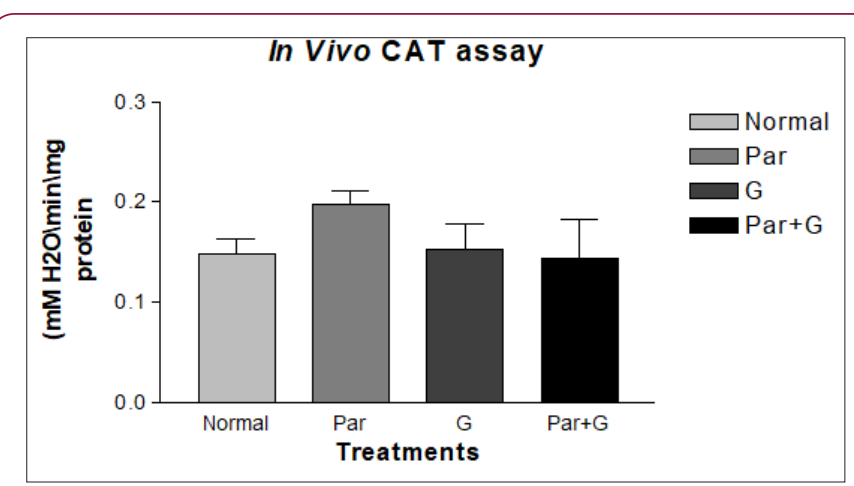

Figure 11: Modulatory effect $G$ on the endogenous markers of oxidative stress HP in adult Drosophila melanogaster. Significances were determined by making comparisons between control vs treated group. Where $\left({ }^{*} \mathrm{p}<0.05\right)$ for the groups treated for $\mathrm{G}^{*}$ with the control group and Par+ $\mathrm{G}^{*}$ with Par.
The results obtained in 'Paraquat Resistance Assay' suggest that G prophylaxis has the propensity to protect against neurotoxicant exposure largely due to its antioxidative potential. In this model, the protective activity of $\mathrm{G}$ was highly comparable to paraquat alone treated. More importantly this data further confirms the utility value of Drosophila system as a primary model to rapidly screen potential compounds for their antioxidant properties prior to their testing in mammalian models and final therapeutic use in humans.

\section{Conclusion}

It is evident from the experimental results that, the leaf extract of sida glutinosa $G$ is good antioxidant, antimicrobial against tested organisms and neuroprotective agent in Drosophila model system. This work opens an avenue for further investigation towards the isolation characterization of active principle and evaluation of biological properties in higher model system. In addition, the biological activity of this ethno-medicinal plant justifies its practice by tribal community at B.R. Hills. Further, the active principle needs to be isolated and the lead molecule must be confirmed using specific biophysical techniques.

\section{References}

1. Finkel T, Holbrook NJ (2000) Oxidants, oxidative stress and the biology of ageing. Nature, 408(6809): 239-247.

2. Ravindra PS, Shashwat S, Suman K (2004) JIACM. 5: 218-225.

3. Sittipunt C (2005) Paraquat poisoning. Respir Care 50(3): 383-385.

4. Suntres ZE (2002) Role of antioxidants in paraquat toxicity. Toxicol 180(1): 65-77.

5. Smith LL, Rose, M S, Wyatt I (1978) The pathology and biochemistry of paraquat. Ciba Found Symp 65: 321-341.

6. Tapiwanashe M, Melanie W, Kumars R, Michael PM, Robin AJ (2006) Mech Age Devpt 127: 356-370.

7. Fryxell PA (1988), Syst Bot Monogr 25: 391.

8. Das N, Achari B, Harigay Y, Dinda B (2011) A new flavonol glucoside from the aerial parts of Sida glutinosa. J Asian Nat Prod Res 13(10): 965-997.

9. Akilandeswari S, Senthamarai R, Valarmathi R, Prema S (2010) Screening of Gastric Antiulcer Activity of Sida acuta Burm. Int J Pharm Tech Res 2: 585-587.

10. Indian drug manufacturers Association. Indian herbal Pharmacopeia Revised new edition (2002) Mumbai, India: Indian drug manufacturers Association.

11. Harbourne (1973) Methods of extraction \& isolation. In: Phytochemical methods Chapman \& Hill, London.

12. Trease GE, Evans WC (1989) Comparative Phytochemical and Nutritional Composition of Trichosanthes cucumerina (L.) and Some Solanum lycopersicum (L.) Cultivars in Nigeria Pharmacognosy $13^{\text {th }}$ Ed Bailliene Tindall Ltd London.

13. Nada MA, Hamdi MH, Nadia GK, Omar AM (2008) Molecules 13: 15011517.

14. Sarojini BK, Darshan Raj CG, Ramakrishna MK, Ramesh SR, Bharath BR, et al. (2011) Lett in Drug Des \& Disc 8: 260-267.

15. Ravikumar H, Muralidhara (2009) Neuro Toxicol 30: 977-985.

16. Lin A (1998) Extended life-span and stress resistance in the Drosophila mutant methuselah Science 282(5390): 943-946. 
17. Ohakawa H, Ohishi U, Yakgi K (1979) Assay for lipid peroxides in animal tissues by thiobarbituric acid reaction. Anal Biochem 95(2): 145-149.

18. Wolf, SP (1994) Ferrous ion oxidation in presence of ferric ion indicator xylenol orange for measurement of hydroperoxide. Meth Enzymol 233: 182-189.

19. Hissin PJ, Hilf R (1976) A fluorometric method for determination of oxidized and reduced glutathione in tissues. Anal Biochem 74(1): 214216.

20. Aebi H (1984) Catalase in vitro. Meth Enzymol 105: 121-126.

21. Kakkar BD, Viswanathan PN (1984) A Modified Spectrophotometric Assay of Superoxide Dismutase. Ind J Biochem Biophys 21: 130-132.

22. Lowry OH, Rosebrough NJ, Farr AL, Randall RJ (1951) J Biol Chem 282: 943-946.

\section{ISSN: 2574-1241}

DOI: 10.26717/BJSTR.2018.11.002171

Panduranga Murthy G. Biomed J Sci \& Tech Res

(c) (i) This work is licensed under Creative

Submission Link: https://biomedres.us/submit-manuscript.php
23. Chaudhury A, Bowling $\mathrm{K}$, Funderburk $\mathrm{C}$, Lawal $\mathrm{H}$, Inamdar A et al. (2007) J Neurosci 27: 2457-2467.

24. Feany M B \& Bender W W (2000) A Drosophila model of Parkinson's disease. Natr 404: 394-398.

25. Orr WC, Radyuk SN, Prabhudesai L, Toroser D, Benes JJ, et al. (2005) J Biol Chem 280: 37331-37338.

26. Thiruchelvam M, McCormack A, Richfield E K, Baggs RB, et al. (2003) Age-related irreversible progressive nigrostriatal dopaminergic neurotoxicity in the paraquat and maneb model of the Parkinson's disease phenotype. Eur J Neurosci 18(3): 589-600.

27. McCormack AL, Atienza JG, Johnston LC, Andersen JK, Vu S, et al. (2005) Role of oxidative stress in paraquat-induced dopaminergic cell degeneration. J Neurochem 93(4): 1030-1037.

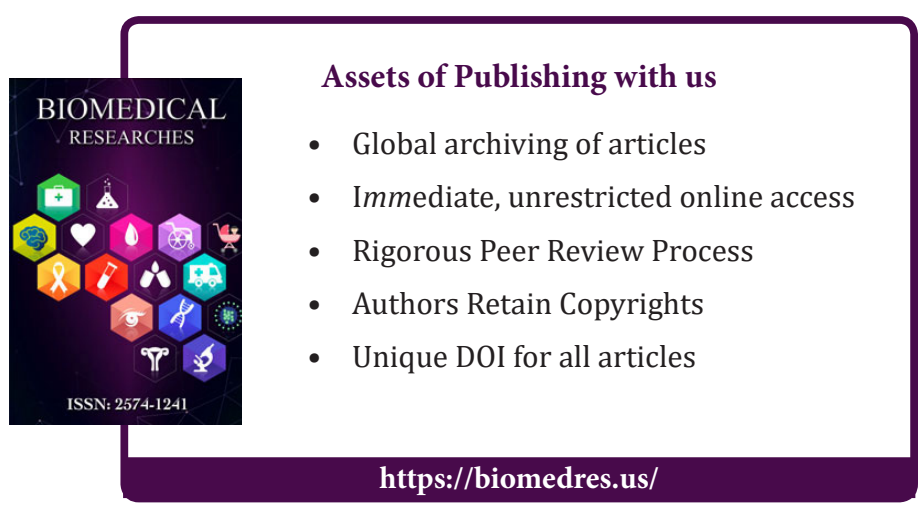

